\title{
Representaciones sociales de niñas y niños acerca de su barrio en un contexto de narcotráfico ${ }^{1}$
}

\author{
Roxana Loubet Orozco²; Emilia E. Sánchez Arellano ${ }^{3}$; Cristian D. Torres Osuna ${ }^{4}$; Giova Camacho Castro 5
}

Recibido: 25 de febrero de 2020 / Aceptado: 19 de junio de 2020

Resumen. Se presenta parte de los resultados de una investigación que tuvo como objetivo analizar las representaciones sociales de niños y niñas de un asentamiento con actividades ligadas al narcotráfico en una ciudad ubicada en el Pacífico mexicano. Fue una investigación de tipo cualitativo, teniendo como premisa de partida el que las representaciones de los niños acerca de la vida social se relacionan con experiencias de socialización múltiple. Como estrategia metodológica se realizó un taller de dibujo en el que participaron 5 niños y 6 niñas, de entre 7 y 12 años de edad. Entre los principales hallazgos se observó la importancia de la familia y la cercanía con los vecinos como factores mediadores en la fuerza de las representaciones sociales.

Palabras clave: Representaciones sociales; socialización; infancia; narcotráfico.

\section{[pt] Representações sociais de meninas e meninos sobre seu bairro em um contexto de tráfico de drogas}

Resumo. Se apresenta parte dos resultados de uma investigação que teve como objetivo analisar as representações sociais de crianças em um assentamento com atividades relacionadas a drogas em uma cidade localizada no Pacífico mexicano. Foi uma pesquisa qualitativa, baseada na premissa de que as representações das crianças sobre a vida social estão relacionadas a experiências de socialização múltipla. Como estratégia metodológica foi realizada uma oficina de desenho na qual participaram 5 meninos e 6 meninas, entre 7 e 12 anos. Dentre os principais achados, observou-se a importância da família e a proximidade com os vizinhos como fatores mediadores da força das representações sociais.

Palavras chave: Representações sociais; socialização; infância; narcotráfico.

\section{[en] Social Representations of Girls and Boys on their Neighborhood in a Context of Drug Trafficking}

Abstract. Part of the results of an investigation that aims to analyze the social representations of boys and girls from a settlement with activities related to drug trafficking in a city located in the Mexican Pacific is presented. It was a qualitative research, having as a starting premise that children's representations of social life are related to multiple socialization experiences. As a methodological strategy, a drawing workshop was held in which 5 boys and 6 girls, between 7 and 12 years old, participated. Among the main findings, the importance of family and closeness to neighbors were observed as mediating factors in the strength of social representations.

Keywords: Social representations; socialization; childhood; drug trafficking.

Sumario. Introducción: infancia y narcotráfico en un barrio sinaloense 2 . Referentes teóricos. Socialización y representaciones sociales. 2.1. Socialización de niños y niñas. 2.2. Representaciones sociales. 3. Notas metodológicas. 3.1. Taller de soportes

\footnotetext{
Los resultados que se presentan en este artículo forman parte de una tesis de grado de master en Ciencias Sociales realizada con el apoyo del programa de becas nacionales del CONACYT-México.

Facultad de Ciencias Sociales, Universidad Autónoma de Sinaloa, Mazatlán, México.

E-mail: roxloubet@uas.edu.mx

Universidad TecMilenio. Campus Mazatlán, Sinaloa, México.

E-mail: are32 ailime@msn.com

4 Facultad de Ciencias Sociales, Universidad Autónoma de Sinaloa, Mazatlán, México.

E-mail: pinycity@hotmail.com

5 Facultad de Ciencias Sociales, Universidad Autónoma de Sinaloa, Mazatlán, México.

E-mail: giova3791@hotmail.com
}

Soc. Infanc. 4, 2020: 55-67 
gráficos (dibujos). 3.2. Población. 4. Representaciones sociales de niñas y niños acerca de su barrio. 4.1. El barrio. 4.2. La gente del barrio. 4.3. La familia. 5. Discusión y Conclusiones. 6. Referencias bibliográficas.

Cómo citar: Loubet Orozco, R.; Sánchez Arellano, E. E.; Torres Osuna C. D.; Camacho Castro, G. (2020): Representaciones sociales de niñas y niños acerca de su barrio en un contexto de narcotráfico, Sociedad e Infancias, 4, 55-67.

\section{Introducción: infancia y narcotráfico en un barrio sinaloense}

El concepto de infancia, al igual que otros que relacionamos con la edad, como juventud, adulto, vejez o tercera edad, son conceptos construidos socialmente, pues su significado tiene que ver con el contexto sociohistórico y político en el que se insertan.

No obstante, desde una perspectiva estructural (Qvortrup, 2010a, 2010b) hablar de infancia implica estudiarla como una categoría sociológica permanente, y en este sentido como un colectivo con características únicas y distintivas de otras categorías. Para Qvortrup (2010b) esta distinción puede constituirse a partir de la posición social que como grupo tienen en relación a otro dentro de la estructura generacional, independientemente de su condición de clase, sexo, etnia, etc., aunque, al mismo tiempo, no se puede perder de vista que existen diferencias entre los niños y las niñas acorde a las condiciones objetivas y subjetivas en las que se desenvuelven.

Sostenidos en este marco general, y partiendo de la idea de que niños y niñas son capaces de representarse realidades del orden social y construir teorías que dan sentido a su mundo (Delval, 1989), surgió la inquietud de conocer aquellas elaboradas por niños y niñas de una colonia ${ }^{6}$ o conjunto habitacional de clase popular, donde desde el conocimiento público se desarrollan actividades delictivas asociadas al narcotráfico, en la ciudad de Mazatlán, Sinaloa, México.

En un primer momento se consideró estudiar las representaciones sociales (RS) acerca del narcotráfico, dado que en general, por la evolución sociohistórica que ha tenido el fenómeno en esta parte del país, se ha observado que en el imaginario de los sinaloenses (adultos) el tráfico de drogas no constituye un problema grave como tal (Moreno, et al., 2013; Moreno, Burgos y Valdez, 2016), pudiendo llegar a idealizar a los protagonistas como protectores de la población civil ante la incompetencia de las autoridades. Empero, al realizar el estudio dentro del asentamiento y con el fin de evitar riesgos, las preguntas de investigación se orientaron hacia la vida en el barrio.

En México, de acuerdo con el censo de 2015, los niños y niñas (de 0 a 17 años) representan un tercio del total de la población, distribuyéndose proporcionalmente entre hombres y mujeres (50 y 50), 62\% habitando en zonas urbanas y el $38 \%$ en localidades rurales; la mayoría habita en hogares con alguno de sus padres, pero más de la mitad vive en condición de pobreza (UNICEF, 2018). Aunada a la condición de desigualdad económica, en la última década se han intensificado manifestaciones de violencia asociada principalmente al fenómeno del narcotráfico afectando a la población infantil, sobre todo en la región del noroeste de la República, donde se encuentra ubicada la ciudad de Mazatlán.

En las dos últimas décadas ha aumentado considerablemente la tasa de homicidios dolosos de población infantil como víctimas de las actividades del crimen organizado y tráfico de estupefacientes, especialmente en Baja California, Chihuahua, Durango y Sinaloa (Geremia, 2011; Álvarez y Castillo, 2019). De acuerdo con datos oficiales, de 2006 a 2010 se detuvo a 3.664 menores de 18 años en operativos federales contra la delincuencia organizada (Geremia, 2011), en tanto que, en 2011, se detuvo a 1.044 por delitos contra la salud, homicidio, tráfico de drogas y portación de arma de fuego (Cisneros (2014).

Existe información de que los jóvenes que participan en las filas del crimen organizado lo hacen como parte del grupo armado (Cisneros (2014), así como en actividades de tráfico de droga y de personas, cobro de piso, extorsión, piratería, asaltos, secuestros y asesinatos (CIDH, 2015; Infoabe, 2019). De acuerdo con la Comisión Interamericana de Derechos Humanos (CIDH, 2015) los niños son integrados en organizaciones criminales a partir de los 10 y 11 años de edad, o incluso a edades más tempranas; los más pequeños suelen ser utilizados como vigías o informadores, los de entre 12 y 15 años cuidan las casas de seguridad y los más grandes, a partir de los 16 años, se involucran en secuestros, y asesinatos. En la actividad del narcotráfico específicamente, según el mismo informe de la CIDH (2015), los niños -varones- se encuentran en toda la línea de la industria distribuidos en diferentes roles de acuerdo a su edad: a los más pequeños se les encuentra como vigilantes; con unos años más se involucran en el traslado de la droga y a partir de los 16 años son utilizados como sicarios; en tanto que a las niñas se les incorpora sobre todo en el empaquetamiento de la droga.

\footnotetext{
En México el término colonia se refiere a los barrios en que se constituyen las ciudades, se define como "cada una de las zonas urbanas que se forma alrededor del centro de una ciudad" (Diccionario del español de México (DEM). El Colegio de México, A. C., https:/dem.colmex.mx); se usa además los términos "colonia popular" para referirse a un asentamiento producto de invasiones o subdivisiones ilegales (Hernández, 2005) que ha sido formalizado y que es habitado generalmente por personas de clase baja y media baja; y "colonia residencial" o "fraccionamiento" para las zonas que de antemano han sido planeadas y equipadas con infraestructura y servicios urbanos, las cuales son comúnmente habitadas por familias de clases medias y altas.
} 
La adhesión de menores de edad en las organizaciones criminales se asocia a la desigualdad, pobreza y exclusión social. Los niños y niñas de comunidades pobres y marginadas, con hogares violentos, pueden ser los más vulnerables y presas fáciles de cooptar por las redes del narcotráfico, no solo porque les ofrece empleo, estrategia de inserción y ascenso social, sino también porque les otorga un sentido de pertenencia (Nájar, 2011), afectando negativamente sus oportunidades de desarrollo y el ejercicio de sus derechos (Reyez, 2017).

En el caso del sitio de nuestra investigación, los primeros acercamientos exploratorios mediante observación directa, entrevistas informales y revisión de notas de prensa local mostraron que cada día, a la luz de todos los residentes, se lleva a cabo la venta y consumo de drogas, particularmente, en un callejón por donde solo se internan vecinos y compradores de droga, además de la participación de menores de edad como vigilantes para alertar a los vendedores cuando se da la presencia de autoridades encargadas de la seguridad pública.

La colonia es popularmente conocida por sus particulares callejones un tanto laberínticos donde cualquiera puede perderse o escaparse, pero también por el imaginario social de que en ella impera "la perdición" y el abandono de las autoridades. Es percibida como una zona peligrosa, un lugar al que la gente ajena teme introducirse por las historias sobre delincuencia y venta de droga.

Cabe mencionar que, a pesar de ser un mismo barrio, se puede dividir en dos grandes espacios de actividad delictiva: una de tráfico y consumo de droga y otra de robo a casa habitación.

El espacio que le ha dado la identidad de un lugar de alto riesgo es el de los callejones donde se vende droga, actividad en la que están inscritos adolescentes y jóvenes de entre 14 y 25 años que viven en este mismo barrio, además de algunas mujeres que se encargan de la administración de las casas en donde guardan la droga y de la colocación de la misma en los puntos, así como de vigilar y avisar si se acercan patrullas policiacas, desenvolviéndose en el rol de "halcones" o vigilantes.

Los niños que viven en el callejón principal donde se vende droga asisten a una escuela cercana de 8 de la mañana hasta las 4 de la tarde, pero al regresar a sus casas, tanto niñas como niños, mientras juegan en la calle se desenvuelven también como "halcones", avisando de la presencia de autoridad cercana a la colonia; la diferencia con los adultos es que ellos no están aún en las nóminas del narco, lo hacen porque lo han interiorizado de esa manera, como una reacción "natural". Cuando los niños se cansan de jugar, se sientan en la esquina del callejón sobre las escaleras de caracol de una casa; desde ahí avisan a los vendedores si la autoridad anda cerca con expresiones tales como "por arriba", "por abajo".

La otra zona del asentamiento se encuentra en calles y callejones aledaños, hacia el centro y sur de la colonia; no hay presencia de venta de drogas, sin embargo, son frecuentes los robos en casas, efectuados por jóvenes adictos a las drogas, que, según los propios vecinos, ante la falta de dinero para el consumo, violan la ley y roban a sus vecinos, en lugar de buscar un empleo formal y legal.

En esta parte los niños no fungen como "halcones", pues a ellos sus padres prefieren vigilarlos cuando salen a la calle a jugar o mantenerlos en sus casas, viendo videos en internet o navegando en las redes sociales, pero saben lo que ocurre en el otro lado de la colonia y conviven cotidianamente en ese ambiente.

El barrio estudiado, ${ }^{7}$ situado en las inmediaciones portuarias de la ciudad de Mazatlán, se originó como producto de una invasión de parte de familias de pescadores que fueron desalojadas de otras áreas urbanas alrededor de 1936. Debido a esto, la colonia creció como un conjunto habitacional marginal, con viviendas construidas por los mismos habitantes en suelos inadecuados y sin acceso por años a los servicios públicos elementales y en lotes sin la debida legalización.

A pesar de su origen, no es un área habitacional en extrema pobreza y es catalogada como de bajo rezago social; es una colonia popular, donde conviven familias de ingresos medios y de hasta muy bajos recursos -económicos, sociales y simbólicos-, por lo que se puede clasificar, en general, como una colonia de clase media baja y clase baja. Ubicada en la zona urbana, de acuerdo con datos del censo de 2010 (INEGI, 2010), cuenta con todos los servicios públicos y la mayoría de sus viviendas, acondicionadas con los bienes indispensables, tienen 3 cuartos o más.

No obstante, por sus características en la composición de hogares -alto porcentaje de hogares con jefatura femenina y promedio de hijos por mujer similar al de zonas rurales-, baja escolaridad y escasez en bienes digitales, empleos inestables y de bajos ingresos, alta proporción de personas sin derechos de salud, aunado a un alto índice de actividad delictiva y deterioro urbano, la colocan en condición de vulnerabilidad social.

Por lo anterior, el interés de la investigación se centró en analizar las representaciones sociales que los niños y las niñas residentes de este barrio construyen a partir de su interacción cotidiana en este escenario delictivo. Si bien, se parte de una posición crítica en torno a las teorías funcionalistas de socialización que conciben a la infancia como entidad pasiva, no dejamos de lado que los niños y niñas se constituyen en un contexto relacional múltiple (Lahire, 2007) y, en ese sentido, intersubjetivamente, considerando el espacio social en el que se encuentra la familia, el barrio, la escuela, los medios, además de sus pares y el mundo adulto en general. Siguiendo a Delval (1989) se parte de que las ideas del mundo social de niños y niñas son reconstrucciones y no un mero reflejo de lo que piensan o transmiten los adultos. Así, en la línea de Sirota (1998), para comprender a la infancia ya no cabe preguntarse acerca de lo que produce la familia, la escuela o el Estado, sino más bien qué es lo que producen los niños y las niñas en la interacción con las diferentes agencias de socialización.

Datos tomados de texto inédito elaborado y proporcionado por Enrique Vega Ayala, cronista oficial de la ciudad de Mazatlán. 
De ahí pues, que se propuso como pregunta de investigación: ¿Qué representaciones sociales acerca de la vida en el barrio construyen los infantes en un contexto de socialización de alto riesgo, ligado a las actividades de narcotráfico? y como supuesto de partida, el que las representaciones de los niños acerca de la vida social se relacionan con experiencias de socialización múltiple; en ese sentido, un contexto público como el barrio, ligado a actividades ilícitas y o violentas, funge como escenario de socialización, mediado principalmente por otros procesos de interacción en el seno de la familia, la escuela y por la cultura de pares.

\section{Referentes teóricos: Socialización y representaciones sociales}

\subsection{Socialización de niños y niñas}

Hablar de socialización es referirse a un proceso que no es meramente individual puesto que se constituye en la interacción social. De manera general, se define como un proceso a partir del cual "un ser biológico se transforma en un ser social propio de una sociedad determinada" (Lahire, 2007: 25); un proceso en el cual los individuos incorporan normas, roles, valores, actitudes y creencias, insertos en un contexto socio-histórico y a través de diversos agentes de socialización como la familia, los grupos de pares, los medios de comunicación y otro tipo de instituciones como las educativas, religiosas, recreativas, entre otras (Simkin y Becerra, 2013).

Siguiendo una perspectiva crítica, concordando con Gaitán (2006), cabe enfatizar que los procesos de socialización son bidireccionales y los niños y niñas protagonistas en la construcción de conocimiento, en tanto se les reconoce como actores o como agentes sociales; los niños y niñas son capaces se recrear, innovar o subvertir el orden social, ya que, como actores sociales, "despliegan diversas formas de vivir sus infancias, por lo tanto, los modos en que se reproduce el orden social también cambian” (Pavez, 2012: 85). En ese sentido, con Delval (1989) se asume que en la infancia se realizan tareas de elaboración personal, a partir de lo cual el niño se reconstruye organizando peculiarmente la información que le rodea, en tanto que para Corsaro (2011) se da un proceso de reproducción interpretativa en el que niños y niñas no solo internalizan los saberes sociales y culturales, sino que también contribuyen en la producción y cambio cultural.

Además, en los tiempos de las tecnologías digitales y veloces cambios sociales, las generaciones más antiguas también van aprendiendo e interiorizando nuevos conocimientos y maneras de ver el mundo acorde a los significados de las nuevas generaciones, por ejemplo, cuando los abuelos acuden a sus nietos buscando ayuda para navegar en Internet o manejar el teléfono móvil, o cuando los padres se ven enfrentados en sus prácticas por los reclamos infantiles sobre cuidado del medio ambiente.

En ese tenor, se observan los procesos de socialización como un factor que interviene en la constitución de las representaciones de la vida social, pero en relación a los contextos y particularidades de la infancia. Es decir, no como una determinación lineal que marcarán la vida adulta, sino como procesos que habilitan a niños y niñas como actores sociales que son.

Según Lahire (2007), es necesario pensar la socialización en la infancia en términos de interdependencia entre los escenarios y actores que comprenden su mundo, es decir, la familia, la escuela y los pares; y al que habría que agregar el barrio. Las formas que toman las relaciones de interdependencia con las personas que le rodean de modo más habitual y duradero influyen en la configuración de las disposiciones mentales y comportamentales, en ese sentido, no se da una reproducción fiel de las maneras de actuar de su entorno, "sino que conforma su propia modalidad de comportamiento en función de las diferentes configuraciones sociales en las que está inserto" (Lahire, 2007: 24).

Desde este punto de vista, se considera a los niños como sujetos plenamente sociales, formándose en relación con distintos campos societales, sean políticos, económicos, ocupacionales, educacionales, etc., donde la familia de origen se encuentra en el centro de las relaciones, como un intermediador relevante, aunque no restringido solo a las relaciones interpersonales (como lo hacen algunos enfoques psicológicos, por ejemplo), ya que las familias concretas se ubican en un campo históricamente estructurado y cambiante. La familia sigue teniendo un peso importante en la socialización, aun en las sociedades complejas debido a la carga emocional de los roles parentales (Berger y Luckmann, 1996), y "porque la identificación del joven con sus padres constituye la primera y tal vez la más decisiva de las identificaciones de la personalidad virgen del niño" (Michel, 1991: 85).

Como parte de la etapa de socialización secundaria, la escuela resulta tan importante como la familia en tanto que las dos expresan un sentido de autoridad, pero mientras que en la familia se funda en el afecto y la dependencia, en la escuela la autoridad viene directamente de la sociedad, legitimada por la lógica del saber y con la fuerza legal necesaria para hacerlo cotidianamente durante una gran parte significativa de la vida de los niños y niñas (Fernández Enguita, 1991).

El proceso socializador escolar se da en un espacio de relaciones sociales institucionalizadas con una función integradora al preparar a los infantes para introducirse en la vida social en su conjunto, interiorizando normas y valores de la sociedad civil y del Estado (Durkheim, 1976); contribuye a la diferenciación otorgando las herramientas conceptuales acorde a los grupos de pertenencia profesional (Durkheim, 1976), y a partir de la competencia en el sistema de calificaciones (Parsons, 1976); contribuyendo con lo anterior en la reproducción de la estructura de clases sociales (Bourdieu y Passeron, 1996), pero con posibilidad de generar espacios de prácticas contestatarias y de resistencia (Giroux, 2004). 
En los procesos de socialización durante la infancia destaca también la presencia de las culturas pares, las cuales forman parte de las experiencias que se comparten con otros iguales y que no tienen que ver con la madurez o el desarrollo individual, sino que forman parte de su historia como miembros activos de una sociedad dada (Gaitán, 2006). Por cultura par se entiende un conjunto de rutinas, objetos, valores e inquietudes que se producen y se comparten en interacción con otros iguales (Corsaro y Eder, 1990), a partir de lo cual se da un proceso de apropiación creativa de la información que proviene del mundo adulto, como reproducción interpretativa que se produce de manera única en la cultura par (Corsaro, 1992).

Por otro lado, el barrio se concibe como un espacio social básico de sociabilidad donde se pueden conocer las normas generales y particulares de la convivencia ciudadana (Galindo, 1992). Se considera el gran mediador entre el mundo privado de la casa y el mundo público de la ciudad, un espacio donde se generan nuevas mezclas o hibridaciones de identidades culturales (Koldorf, de Castro y López, 2008). En este sentido, el barrio también es espacio de inscripción cultural, introduce al habitante en una red de signos sociales ya existentes, por lo que, de acuerdo con Ruiz (2002), el barrio se construye como territorio a través de las acciones de los ciudadanos con miras a establecerlo, consolidarlo y defenderlo, "lo mismo que a través de las huellas y claves de marcación y demarcación que establezcan en él con el fin de particularizarle" (Ruiz, 2002: 31).

La práctica en el barrio permite el reconocimiento del espacio como espacio social, donde cada uno tiene su lugar, dando fe de un origen e insertándose "en la historia del sujeto como la marca de una pertenencia indeleble en la medida en que es la configuración inicial, el arquetipo de todo proceso de apropiación del espacio como lugar de la vida cotidiana pública" (Mayol, 1999:11).

En Latinoamérica y particularmente en México, el espacio público es un espacio primario de interacción cotidiana, siendo principalmente en las zonas o colonias populares donde se observan con mayor frecuencia estrategias de producción y consumo de este espacio (Hernández, 2005), así como interacciones de solidaridad y de apoyo social entre las familias que habitan estos hábitat (Guerrero y García, 2012).

\subsection{Representaciones sociales}

Serge Moscovici (1979) fue el creador del concepto de Representaciones Sociales (RS), partiendo, principalmente, de rescatar la idea de representación colectiva de Durkheim, en la que se establece como base que lo colectivo no se reduce a lo individual. No obstante, Moscovici, a diferencia de Durkheim, propone que las representaciones sociales no sólo reproducen las formas de conciencia que la sociedad impone a los individuos, sino que implica un proceso activo de producción y elaboración por parte de los individuos, además de que, para Moscovici (1979), dadas las características de las sociedades actuales, no se puede hablar de representaciones homogéneas compartidas por todos los miembros que conforman la sociedad.

Una de las críticas que se han hecho a la teoría de las RS es que no existe una definición estática del concepto, sin embargo, las aportaciones de diversos autores están encaminadas a que éstas corresponden a un acto de pensamiento en el que un sujeto se relaciona con un objeto y mediante diversos mecanismos de apropiación e interpretación el objeto queda representado simbólicamente en la mente del sujeto. Esta teoría permite trascender el determinismo social, en tanto que se comprende la perspectiva de los agentes en el marco de un contexto social, histórico y cultural; posibilita acceder al mundo propio de los agentes, rescatando su voz e interpretándola en el contexto sociocultural donde se genera (Mireles, 2011).

De acuerdo con López (2009) las RS sirven para poder entender el mundo, su elaboración, selección de la información y el levantamiento de la información conlleva intereses específicos y necesidades concretas de cada grupo. Para Piña y Cuevas (2004) (retomando a Jodelet), la noción de representación social surge en un primer momento como un concepto para explicar diversos fenómenos de las interacciones sociales de la realidad cotidiana, por lo que es importante observar los procesos de construcción de los objetos de representación en contextos específicos, ya que para Jodelet (1986) las RS no son acerca de todo el mundo social sino sobre algo o alguien en específico y además son expresadas por un sector social particular. No son sobre una sociedad específica ni en un sentido universal sino, por el contrario, sobre objetos, sujetos, ideas o acontecimientos de esa sociedad en particular. Así entonces, las representaciones sociales no son sólo productos mentales; son, además, construcciones simbólicas que se crean y recrean en el curso de las interacciones sociales, dándole al sujeto el papel de constructor de significados.

Para construir las RS se ha de recurrir a las connotaciones simbólicas que nos proporciona el lenguaje desde el discurso que producen los individuos en sus prácticas sociales, ya que "la comprensión del lenguaje es esencial para cualquier comprensión de la realidad de la vida cotidiana" (Berger y Luckmann, 1996: 55). De este modo, las RS son "modalidades de pensamiento práctico orientado hacia la comunicación, la comprensión y el dominio del entorno social, material e ideal" (Jodelet, 1986: 472).

¿Dónde están las RS? Entendidas como formas de pensar y recrear la realidad social, éstas se encuentran en la subjetividad de las personas o sujetos de estudio; en su mundo, su cultura, sus creencias, producidas por la experiencia que los individuos tienen en su vida cotidiana, y sirven como explicación útil de la construcción social de la realidad. Son un recurso oportuno y viable para conocer las formas y los contenidos de la construcción colectiva de la realidad social (Jodelet, 2000). Brindan la oportunidad de conocer y comprender el punto de vista de los agentes de un grupo en un contexto determinado, puesto que permiten acceder al mundo del sentido común de los agentes, escuchar sus voces y tratar de interpretar sus palabras y acciones en el contexto especifico donde se generan. 
La investigación en América Latina y particularmente en México acerca de las RS es abundante, aunque escasa en población infantil y más aún cuando se aborda en relación a contextos de riesgo, entendidos como espacios habitacionales o institucionales con características de violencia social, ya sea por vivir en situación de calle, por experiencias vividas o próximas, o percepción de inseguridad cercana debido a manifestaciones de violencia por crimen organizado, narcotráfico o conflictos armados.

Al respecto, localizamos once investigaciones realizadas en las dos últimas décadas, a partir de las cuales creamos tres categorías de inclusión: cuidado (de Colombia, Monsalve, et al., 2016; de Perú, Añaños, et al., 2010), género (de Colombia, Zuluaga, 2018; de México, Hernández, 2006) y estratificación y orden social (de México, Almanza, et al., 2018; Inzunza, 2017; Losano y García, 2015; de Colombia Parra, 2011; Alvarado, Ospina y Luna, 2005; y de Chile, Guerrero y Palma, 2010 y Salgado, 2009).

En síntesis, se desprende de los resultados de estas investigaciones que en las RS que construyen niños y niñas la influencia de las estructuras sociales puede depender de cómo se entrelazan los estereotipos de los medios de comunicación masiva, las relaciones familiares, la escuela y las vivencias personales. Y que, en tanto se vivan procesos de socialización primaria dentro de espacios institucionales como la familia y la escuela que atienden a una estructura social más amplia de normas y valores dominantes -como la de género o de autoridad- éstas resultarán de importancia en la constitución de las RS que se forjan en la infancia, matizando aquellas que se producen en la experiencia directa.

\section{Notas metodológicas}

El estudio tuvo como objetivo general analizar las representaciones sociales de los infantes que viven en un contexto de socialización barrial de alto riesgo en la ciudad de Mazatlán, Sinaloa, México. Fue una investigación cualitativa, utilizando herramientas del método etnográfico, como la observación participante y la conversación informal, además de la técnica de dibujo, como elemento central para el análisis. El artículo solo ofrece parte de los resultados de la técnica de dibujo.

Partiendo de un fundamento ético, hemos omitido el nombre del asentamiento y de los niños y niñas participantes, por un lado, por consideraciones generales de la investigación social anonimizando a los sujetos, y por otro, debido al contexto de riesgo en que se inscriben. Tomamos en cuenta que la participación de infantes en la investigación debe hacerse bajo los principios de los derechos del niño, considerándolos como personas dignas de respeto y capaces de participar activamente en la investigación, en un ambiente seguro y protegido. Debido a ello, el escenario en el que se generaron los dibujos fue dentro del hogar de uno de los niños participantes, en la sección de la colonia donde no se vende droga, y orientando el interés del estudio hacia tópicos del barrio en general con estrategias lúdicas, incluyendo a todos los niños y niñas que quisieron y pudieron participar, con autorización de sus padres.

\subsection{Taller de soportes gráficos (dibujos)}

Para poder aplicar esta técnica de recolección de datos fue necesario organizar un taller de dibujo que permitiera de manera más ágil la asistencia y concentración de los niños y niñas, por lo que, para dar a conocer el taller, se hizo a través de una convocatoria con una semana de anticipación.

El taller se llevó a cabo en una sesión, con una duración de 3 horas, dividiéndose en cinco momentos (Martínez, 2015), en los que se les solicitó a los niños y niñas que realizaran cinco dibujos (1 a la vez).

1. Dibujo con temática preestablecida: para arrancar con las actividades, se les indicó que dibujaran en una hoja un dibujo de su barrio (que es su entorno inmediato, en el que construye y es construido), con elementos que lo identifican.

2. Dibujo del barrio en el futuro: en este momento se le pidió al niño que el dibujo que realizara fuera en cuanto al futuro de su barrio, es decir como desearía que fuera su barrio en un futuro.

3. Dibujo de los niños a futuro: en este tercer momento, se pretendía que el niño se dibujara a sí mismo en un futuro deseado, es decir trabajando de adulto, con una breve descripción escrita y oral de cómo le gustaría o se imaginaba que pudiera ser.

4. Dibujo de un habitante de su barrio: en un cuarto momento, se le solicitó al niño que dibujara a sus vecinos, esto con el objetivo de que el niño plasmara elementos importantes en cuanto a los habitantes de su barrio.

5. Dibujo de su familia: en este último momento, se le solicitó al grupo de niños y niñas que dibujaran a su familia con el objetivo de poder rescatar elementos que nos dieran pauta para comprender el tipo de relación familiar.

Se les proporcionó lápices de colores, plumones y hojas blancas. A lo largo de la elaboración del dibujo se realizó un seguimiento sobre sus representaciones y se tomaron en cuenta sus comentarios.

En este artículo solo se presentan los resultados obtenidos en relación a tres de los momentos que hablan del presente: el barrio, los habitantes del barrio y su familia. 


\subsection{Población}

Partiendo de que la representatividad en los estudios cualitativos no está directamente relacionada con la cantidad y extensión de ciertas características de la población a estudiar, sino que se centra en la reconstrucción de vivencias y sentidos, en el taller de dibujo se tuvo la participación de 11 infantes, 5 niños y 6 niñas, de 7 a 12 años de edad, estudiantes de nivel básico (primaria) y todos y todas viven con sus padres. Ninguno de los niños vive dentro de los callejones donde se vende droga.

\section{Representaciones sociales (RS) de niños y niñas acerca de su barrio}

Como lo hemos propuesto antes, los niños y niñas van construyendo sus RS de la vida social del barrio, principalmente tras los procesos de socialización e interacciones que viven en la intimidad de éste y en el seno familiar. Asimismo, como mencionamos en el apartado metodológico, se describen las RS de los momentos acerca del presente: el barrio, la gente y la familia, por lo que, a continuación, plasmamos los resultados organizados en ese orden.

\subsection{El barrio}

La niñez de este asentamiento representa su barrio como algo muy cercano. Al ser el hogar el primer punto de contacto, el grupo de niños y niñas que participaron presentó el exterior de las viviendas o casas como el elemento más fuerte, seguido de componentes de la naturaleza, sus calles y como elemento especial, el edificio de una industria cervecera, que puede ser observada desde diferentes puntos de la colonia (figura 1).

En general, las características con las que dibujaron las viviendas son muy similares a las casas que habitan y que persisten a lo largo del barrio, por ejemplo, edificaciones de dos pisos con balcones con herrería y al ras de calle; aunque también introdujeron otros rasgos como techos terminados en pico o dos aguas y chimeneas, características que no se ven en este barrio, pero que pueden ser propios de referentes compartidos en otros espacios de socialización, como los textos escolares o medios de comunicación (figura 2).

Además, 10 de los 11 participantes dibujaron elementos de la naturaleza como árboles, mismos que tienen características similares en cuanto a la forma y al tamaño: 5 dibujaron árboles grandes, 3 plasmaron árboles medianos y solo 1 representó un árbol de talla chica; cabe mencionar que la colonia, a lo largo de sus calles, tiene árboles de gran tamaño y de edad antigua que fueron sembrados por sus mismos habitantes (figura 3). Además, 6 de los 11 dibujos, plasmaron elementos urbanos como lo son calles y banquetas y en algunos se pueden observar la presencia de automóviles.

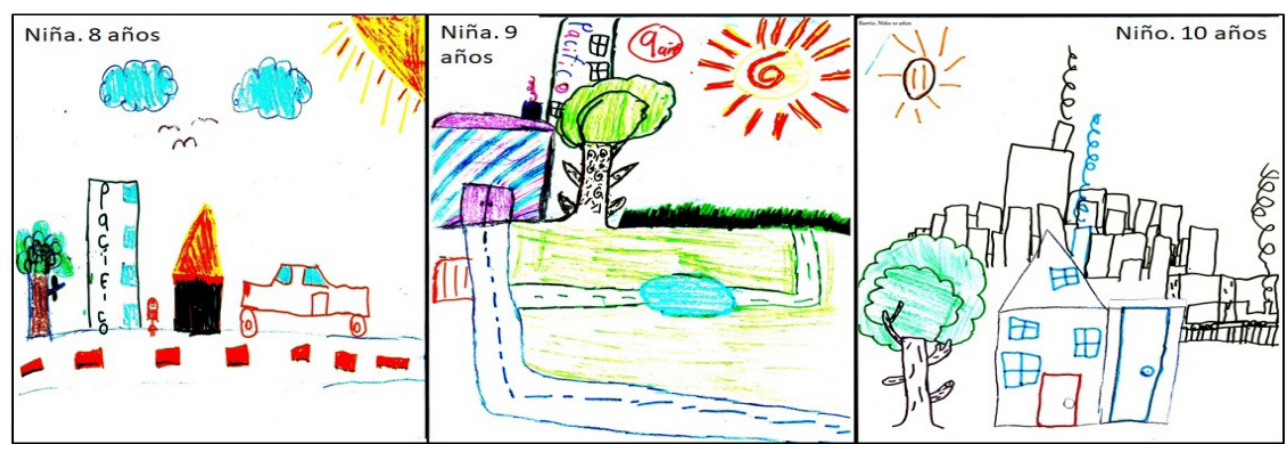

Figura 1. Presencia de la cervecería (edificio alto al fondo). ${ }^{8}$

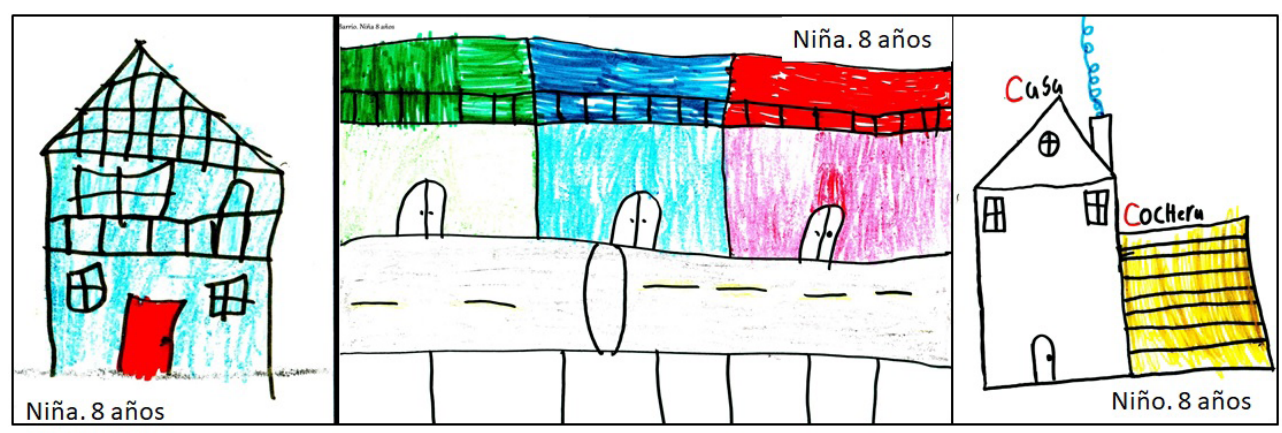

Figura 2. Dibujos de las viviendas del barrio.

En los dos primeros dibujos los niños escribieron el nombre de la cervecería; lo hemos omitido para mantener el anonimato. 


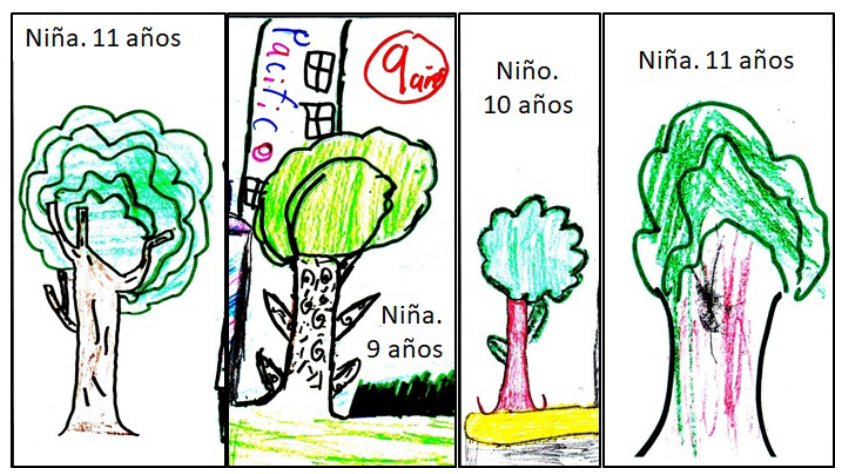

Figura 3. Fragmentos de dibujos de árboles.

Las niñas más grandes, de 10 y 12 años, pudieron representar en su dibujo elementos más específicos y detallados que se pueden encontrar en el barrio, como lo son los elementos religiosos con los que identifican sus calles, al plasmar dibujos de la virgen de Guadalupe, como se puede observar en los gráficos de la figura 4.

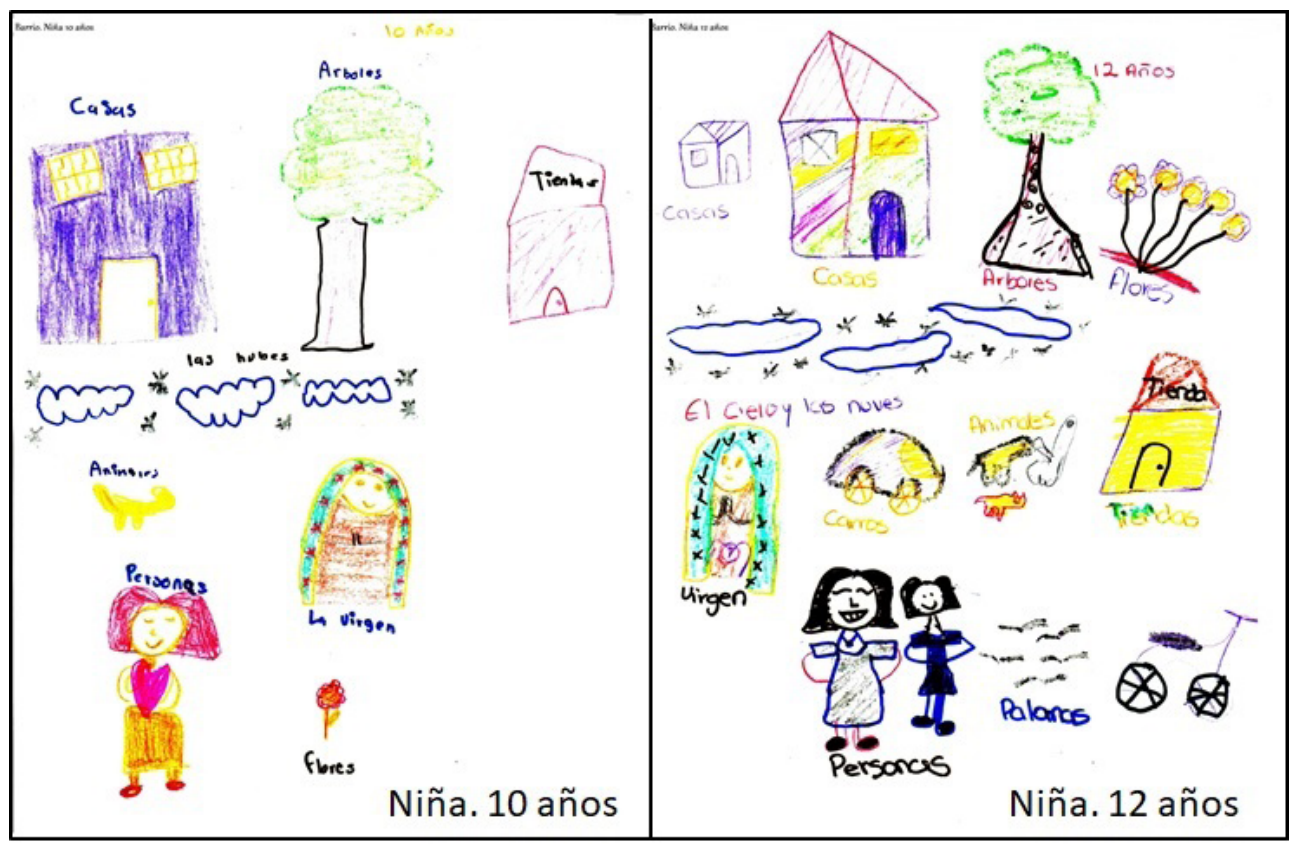

Figura 4. Dibujos de las viviendas del barrio.

La colonia, a lo largo de su dimensión, cuenta con tres murales de vírgenes guadalupanas pintadas por los vecinos y situadas en los puntos más conglomerados en cuanto a habitantes, coincidiendo con la ubicación de los puntos de venta y distribución de drogas. Por otra parte, también dibujaron otros objetos tales como tiendas (abarrotes), animales, bicicleta, flores y personas que muestran gestos de alegría, como parte del entorno vecinal.

En algunos de los dibujos, tanto de niñas como niños, de edad indistinta, se puede observar que los etiquetan, es decir, escriben además de dibujar. Esta característica es denominada por Wright (2007) como intratextualidad, entendida como relaciones dentro del texto para aclarar o mejorar el contenido introduciendo elementos etiquetados, lo que puede ayudar a anclar el texto dentro de un medio.

\subsection{La gente del barrio}

Aunque en el exterior de la colonia se le perciba como un barrio conflictivo y peligroso, los niños y niñas dividen a la gente del barrio en gente "buena" y en "rateros", predominando la que es "buena" (figuras 5 y 6). Por un lado, en 5 de los 11 dibujos, los vecinos aparecen como figuras sonrientes, alegres, algunas unidas y coloridas. En la narrativa, algunos niños hicieron mención de que sus vecinos eran muy "mitoteros" (chismosos), pero que eran gente "amable" y "fiestera". 


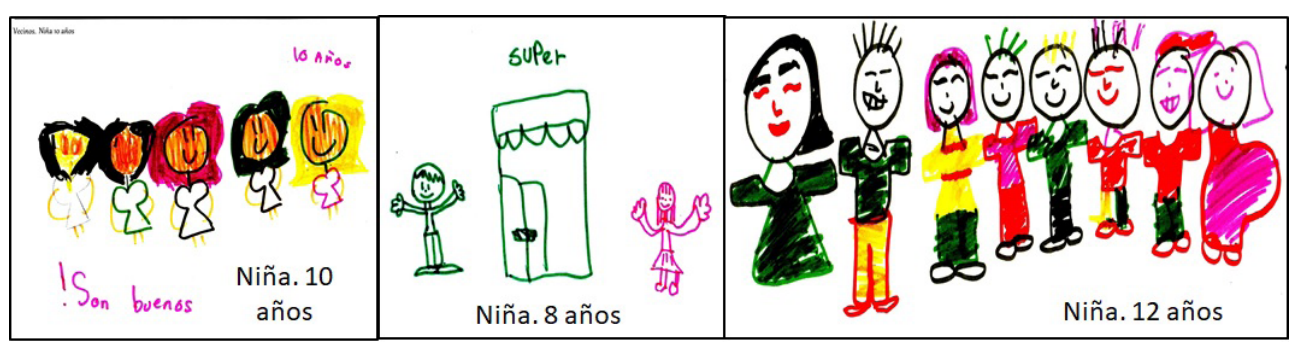

Figura 5. Dibujos de vecinos como "gente buena" o "amable".

Para ellos, la presencia de gente involucrada en las actividades de venta de droga no tiene nada que ver con la característica de la gente amable y fiestera que vive en el barrio. Por el contrario, la gente mala es la que consume droga y roba en sus casas. En 6 dibujos se identifican y narran elementos relacionados con la delincuencia, como lo es la presencia de "rateros", gente fumando marihuana ("con churros de mota", D., 8 años) y con armas de fuego; en cuanto a sus narrativas dijeron que había muchos robos en el barrio y puros drogadictos y marihuanos en las calles, sin embargo, algunos no se atrevieron a dibujarlo y decían: "no, mejor no", notándose una impresión de asociación negativa.

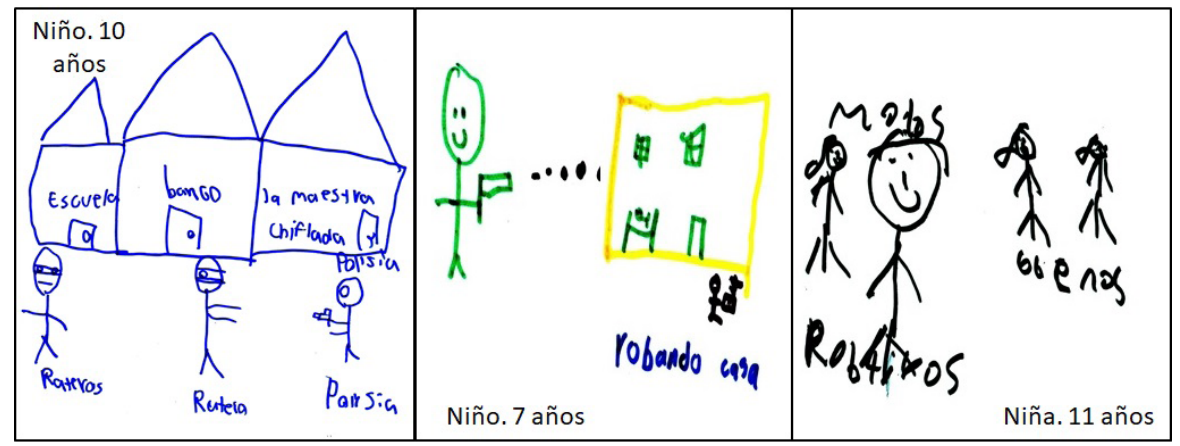

Figura 6. Dibujos de gente "buena" y "mala".

En relación a los dibujos sobre el barrio y los vecinos se observa diferencia en cuanto a la variable de género, ya que, las niñas dibujaron con más atención y detalle su entorno y fueron más profundas en cuanto a la identificación de elementos característicos de su barrio; incluso en la utilización de colores fueron más expresivas que los niños.

Destaca también que todas las niñas dibujaron características relacionadas con lo que para ellas es la "gente buena", a diferencia de los niños que fueron quienes dibujaron personas robando y con armas, etiquetadas como "malas". Es decir, mientras que en la representación de las niñas predomina la gente con la que conviven de manera cercana y perciben como alegre, los niños tienen más presente a aquellos que los perjudican y no temen plasmarlo.

\subsection{La familia}

Todos los niños y niñas participantes representaron a sus familias con semblante de unión y felicidad, lo que nos permite afirmar que tienen lazos familiares fuertes, por lo que el rol de la agencia socializadora de la familia, dentro de la socialización primaria, es importante para ellos, dado que la relación interpersonal del niño, comienza en el círculo familiar.

La presencia de sus familias fue plasmada en los dibujos con familias de 3 miembros y hasta de 10 integrantes. Cabe destacar que 9 de los participantes dibujaron a familias nucleares; además, una extendida y una familia monoparental. En algunos casos realzaron su presencia con el pronombre "yo", a hermanos y hermanas con sus nombres y a los padres con los términos "mamá" y "papá” (figura 7).

Sin pretender un análisis psicológico de los dibujos, se destaca el hecho de que en 4 se presenta la figura de la "mamá" de tamaño más grande que la del "papá", como se puede observar en la siguiente imagen (figura 8). Esto puede significar la fuerte influencia o apego que se tiene hacia este elemento de la familia, principalmente en el caso de las niñas. 


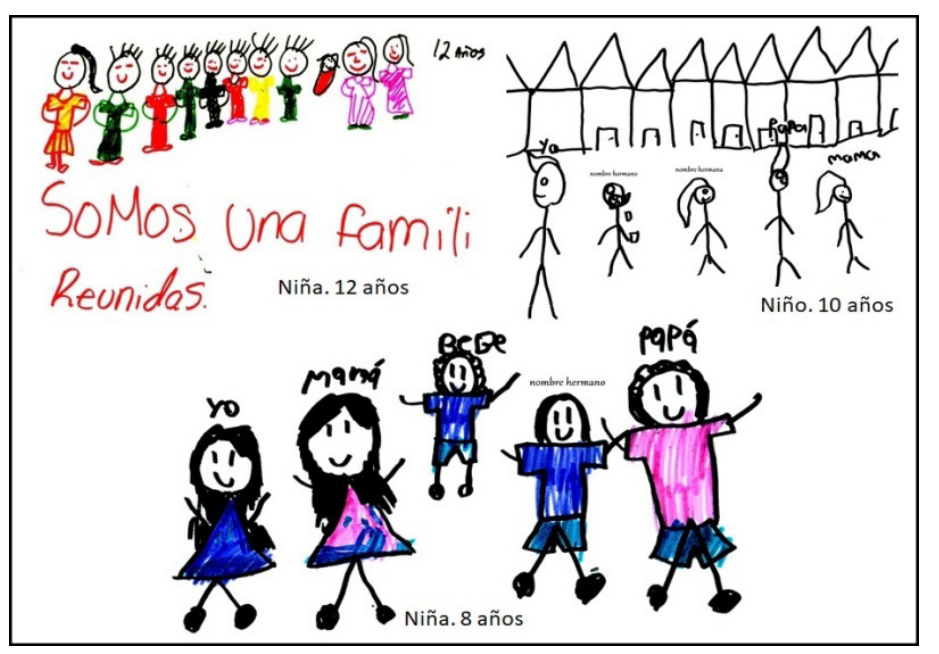

Figura 7. Dibujos de las familias.

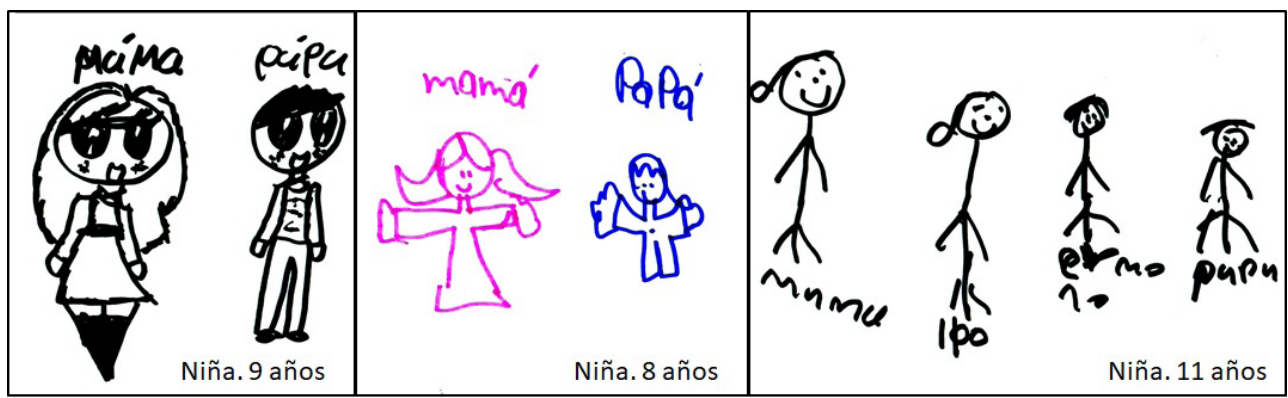

Figura 8. Diferencia en el tamaño de las figuras de "mamá” y "papá”.

\section{Discusión y conclusiones}

En las páginas anteriores se ha evidenciado la presencia de grupos delictivos, vinculados a la acción de grupos dedicados al tráfico de drogas en la colonia objeto de estudio. Existen bandas que tienen años permaneciendo en la zona, controlando y defendiendo el territorio, ganándose, además en parte, el respeto por los vecinos que viven en el asentamiento. Tienen una lógica y estructura organizativa que les permite sustentar el negocio con base en relaciones de alta confianza entre sus miembros y vínculos de amistad con los vecinos.

Pese a la presencia de estas actividades ilegales, la gente no se siente amenazada, aunque para las personas que no viven en este lugar, es un barrio inseguro al que no se atreven ni si quiera a pasar de cerca. Esto mismo se observa en otras investigaciones como las de Oemichen (2013) o Mendoza (cit. en Almanza et al., 2018).

Derivado de la descripción antes expuesta, podemos decir que los niños y niñas participantes, a pesar de vivir en un contexto de prácticas cotidianas relacionadas con el narcotráfico y violencia asociada, no presentan fuertes representaciones sociales ligadas al crimen organizado o prefieren no expresarlas. Si bien reconocen la presencia de "rateros" y de "drogadictos", no se alarman de ello.

En este sentido, y respecto a lo que planteamos en nuestro supuesto hipotético, podemos decir que las RS acerca de las actividades relacionadas con el narcotráfico, específicamente las del narcomenudeo, o las actividades delictivas como los robos a casa habitación, puede estar mediada por los procesos de socialización familiar, en gran medida, figurando como un factor protector. Ya que los padres de los niños y niñas participantes muestran niveles de imposición y control más elevados, a pesar de vivir en este barrio vulnerable socialmente, la atención hacia los hijos es evidente en sus representaciones. Hay que aclarar que pudiera influir la posición de los puntos de venta de droga, ya que como se mencionó a lo largo de la delimitación del espacio, por cuestiones del factor riesgo, no se pudo trabajar con niños que viven en el callejón donde se convive de manera directa y más cercana con el narcomenudeo.

Los procesos de socialización familiar como mediación "determinan la forma en que los contextos residenciales afectan a diversos aspectos del desarrollo psicosocial de niños y adolescentes" (Gracia, Fuentes y García, 2010: 266); estos niños crecen internalizando los elementos de su barrio, pero construyen también a partir de la interacción con la familia y sus pares. Gracia, Fuentes y García (2010) sugieren que los padres que residen en barrios vulnerables o de riesgo muestran niveles de imposición y control más elevados, como sucede en los niños de nuestro estudio.

Cabe destacar, que el barrio aparece como un espacio de vida clave en la conformación de determinadas pertenencias socioterritoriales, pues se podría decir que el barrio, y todo lo que éste implica (interacción con los vecinos, 
actividades cotidianas, lugares alrededor de la vivienda, el entorno), se incorpora en los sujetos al ser una de las principales fuentes de socialización, tan sólo por detrás de la familia.

A lo anterior, podría agregarse la influencia del imaginario social que concibe el narcotráfico como un negocio de tipo económico como cualquier otro, siendo solo los actos violentos los que producen sentimientos de miedo, inseguridad y enojo.

Por otro lado, si bien se observaron algunas manifestaciones relacionadas con el sexo, ya que las niñas dibujaron un entorno más colorido, "bueno" y "feliz", en cuanto a la edad no se registraron diferencias más significativas; esto tal vez, por la pequeña cantidad de sujetos participantes.

Finalmente, se puede concluir que, contrario a lo que se esperaría desde una perspectiva funcional o conductista, las vivencias delictivas observadas por niños y niñas que habitan esta colonia no son absorbidas cual esponjas sin filtro, sino que, en el sentido de la socialización múltiple, y la capacidad de autonomía de la infancia, van construyendo sus representaciones intersubjetivamente.

Es justo anotar también que la limitante del factor riesgo puede ser una oportunidad para que, en futuras investigaciones, se busquen estrategias que permitan ampliar la muestra a niños y niñas que viven y conviven de manera directa con actores que figuran en las filas del narco dentro del asentamiento o de otros en condiciones similares.

Este tipo de investigaciones deben servir para ampliar el conocimiento en torno a la capacidad constructiva y resiliente de la infancia en escenarios de riesgo y fortalecer a través de la política pública aquellos sistemas que intervienen favorablemente en su bienestar, evitando con ello la reproducción de acciones por parte de los gobiernos y de la sociedad civil basadas en estereotipos, que más que ayudar, entorpecen su desarrollo.

\section{Referencias bibliográficas}

Almanza Avendaño, A. M., et al. (2018). Representaciones sociales acerca del narcotráfico en adolescentes de Tamaulipas. Región y Sociedad, 30(72), 1-25.

http://dx.doi.org/10.22198/rys.2018.72.a846.

Alvarado, S.V., Ospina, H. F., Luna, M.T. (2005). Concepciones de justicia en niños y niñas que habitan contextos urbanos violentos. Niñez y Juventud, 3(2), 1-30.

Álvarez Gutiérrez, M. G., Castillo Koshnick, J.G. (2019). Panorama estadístico de la violencia contra niñas, niños y adolescentes en México. México: UNICEF.

Añaños B., F. T., et al. (2010). Realidades y representaciones sociales de las niñas y los niños de la calle de Perú. Revista Española de Drogodependencias, 35(2), 182-194.

Berger, P., Luckmann, T. (1996). La construcción social de la realidad. Buenos Aires: Amorrortu.

Bourdieu, P., Passeron, J. C. (1996). La reproducción. Elementos para una teoría del sistema de enseñanza. México: Fontamara. CIDH (2015). Violencia, niñez y crimen organizado. OEA-Plan International.

Cisneros, J. L. (2014). Niños y jóvenes sicarios: una batalla cruzada por la pobreza. El Cotidiano, (186), julio-agosto, 7-18.

Corsaro, W. A. (1992). Interpretative reproduction in children's peer cultures. Social Psychology Quarterly, 5 (2), 160-177. https://doi.org/10.2307/2786944

Corsaro, W. A. (2011). Sociologia da infancia. Porto Alegre: Artmed.

Corsaro, W. A., Eder, D. (1990). Children's peer cultures. Annual Review of Sociology, (16), 197-220. https://doi.org/10.1146/ annurev.so.16.080190.001213

Delval, J. (1989). La representación infantil del mundo social. En E. Turiel, I. Enesco, J. Linaza (Comps.), El mundo social en la mente infantil (pp. 245-328). Madrid: Alianza.

Durkheim, É. (1976). Educación como socialización. Salamanca: Sígueme.

Fernández Enguita, M. (1991). La escuela a examen. (Un análisis sociológico para educadores y otras personas interesadas). Madrid: Eudema.

Gaitán Muñoz, L. (2006). La nueva sociología de la infancia. Aportaciones de una mirada distinta. Política y Sociedad, 43(1), 9-26.

Galindo Cáceres, J. (1992). Vía pública, vida pública. De los caminos de vida y la calle en la organización urbana. Estudios sobre las culturas Contemporáneas, 4(14), 11-28.

Geremia, V. (2011). Infancia y conflicto armado en México. Informe alternativo sobre el Protocolo Facultativo de la Convención sobre los derechos del niño relativo a la participación de niños en los conflictos armados. México. Red por los Derechos de la Infancia en México.

Giroux, H. (2004). Teoría y resistencia en educación. México: Siglo XXI-UNAM.

Gracia, E., Fuentes, M. C., García, F. (2010). Barrios de riesgo, estilos de socialización parental y problemas de conducta en adolescentes. Psychosocial Intervention, 19(3), 265-278.

Guerrero Barrón, J., García Sánchez, B.Y. (2012). Violencias en contexto. Bogotá: Universidad Distrital Francisco José de Caldas.

Guerrero, P., Palma, E. (2010). Representaciones Sociales sobre educación de niños y niñas de calle de Santiago y Quito. Niñez y Juventud, 8(2), 1025-1038.

Hernández Bonilla, M. (2005). Mejoramiento del espacio público en las colonias populares de México. Caso de estudio de Xalapa-Veracruz. Revista Invi, 20(53), 181-199. 
Hernández Ordóñez, A. (2006). Representación social del género masculino en un grupo de niños y jóvenes que viven en la calle, en la ciudad de México. Segunda parte. Salud mental, 29(1), 56-63.

INEGI (2010). Censo de Población y Vivienda 2010. México: INEGI.

Infoabe (2019, septiembre 12). Los usan y luego los desechan: niños y jóvenes que son carne de cañón del narco. https://www. infobae.com/america/mexico/2019/09/12/los-usan-y-luego-los-desechan-ninos-y-jovenes-que-son-carne-de-canon-del-narco/, acceso 18 de diciembre de 2019.

Inzunza Acedo, B. E. (2017). Los medios de comunicación como fuente de información en la construcción de representaciones sociales de la delincuencia. Comunicación y sociedad (29), 185-201.

Jodelet, D. (1986). La representación social: fenómenos, concepto y teoría. En S. Moscovici, Psicología social II. Pensamiento y vida social (469-494). Barcelona: Paidós.

Jodelet, D. (2000). Representaciones sociales: contribución a un saber sociocultural sin fronteras. En D. Jodelet, A. Guerrero Tapia (Coords.), Develando la cultura. Estudios en representaciones sociales (pp. 7-30). México: UNAM.

Koldorf, A. E., De Castro, R., López Tessore, V. (2008). El barrio como espacio de interacción social. IX Congreso Argentino de Antropología Social. Facultad de Humanidades y Ciencias Sociales - Universidad Nacional de Misiones, Posadas. Recuperado de http://cdsa.aacademica.org/000-080/110.pdf

Lahire, B. (2007). Infancia y adolescencia: de los tiempos de socialización sometidos a constricciones múltiples. Revista de Antropología Social (21), 21-38. https://doi.org/10.5209/rev_RASO.2007.v16.9963

López Gärtner, R. (2009). Representaciones de violencia y paz que los niños y las niñas significan a través de los noticieros de televisión. Tesis de maestría. Centro de estudios avanzados de niñez y juventud. Universidad de Manizales, Colombia.

Losano, M. C., García Horta, J.B. (2015). Representaciones sociales de la educación formal. Un estudio comparativo basado en datos visuales. En A. Méndez Zúñiga (Coord.), Representaciones sociales en ámbitos educativos (pp. 52-77). México: REDIE.

Martínez González, M. (2015). Entre la imagen y la palabra: representaciones infantiles del tejido de palma entre los mixtecos contemporáneos de Santiago Cacaloxtepec. Cuicuilco, 22(64), 245-267.

Mayol, P. (1999). Habitar. En M. De Certeau, L. Girad, L., P. Mayol, La invención de lo cotidiano 2. Habitar, cocinar (pp. 3-132). México: Universidad Iberoamericana/ITESO.

Michel, A. (1991). Sociología de la familia y del matrimonio. Barcelona: Península.

Mireles Vargas, O. (2011). Representaciones sociales: debates y atributos para el estudio de la educación. Sinéctica (36), 1-11.

Monsalve Escudero, L. M., et al. (2016). Representaciones sociales sobre salud en adolescentes habitantes de calle, Medellín 2014. Médicas UIS, 29(3), 33-42. https://doi.org/10.18273/revmed.v29n3-2016003

Moreno Candil, D. M. et al. (2013). Causas a las que atribuyen los culiacanenses el involucramiento de la población de Sinaloa en el narcotráfico. Psico-logos (14), 61-71.

Moreno Candil, D., Burgos Dávila, C. J., Valdez Bátiz, J. E. (2016). Daño social y cultura del narcotráfico en México: Estudio de representaciones sociales en Sinaloa y Michoacán. Mitologías hoy (14), 249-269.

Moscovici, S. (1979). El psicoanálisis, su imagen y su público. Buenos Aires: Huemul.

Nájar, A. (2011, marzo 25). Los “niños soldados” del narcotráfico en México. BBC Mundo (en línea).

http://www.bbc.com/mundo/noticias/2011/03/110324_mexico_ninos_soldados_narcotrafico

Oehmichen Bazán, C. (2011). La violencia de siempre. Representaciones sociales de la violencia delincuencial en un barrio popular de la ciudad de México. Anales de Antropología, 47(1), 243-262. http://dx.doi.org/10.1016/S0185-1225(13)71013-0

Parra Villa, Y. (2011). Representación social del conflicto armado colombiano en niños y niñas de un colegio adscrito a la Policía Nacional. Universitas Psychologica, 10(3), 775-788.

Parsons, T. (1976). La clase escolar como sistema social. Algunas de sus funciones en la sociedad americana. Revista de Educación (242), 64-86.

Pavez Soto, I. (2012). Sociología de la Infancia: las niñas y los niños como actores sociales. Revista de Sociología (27), 81-102. 10.5354/0719-529X.2012.27479

Piña Osorio, J. M., Cuevas Cajiga, Y. (2004). La teoría de las representaciones sociales. Su uso en la investigación educativa en México. Perfiles educativos, 26(105), 102-124.

Qvortrup, J. (2010a). A infância enquanto categoria estrutural. Educação e Pesquisa, 36 (2), 631-643.

Qvortrup, J. (2010b). A tentação da diversidade - e seus riscos. Educação \& Sociedade, 31 (113), 1121-1136.

Reyez, J. (2017, Febrero 26). Los niños de una sociedad criminal. Contralínea (en línea). https://www.contralinea.com.mx/archivo-revista/2017/02/26/los-ninos-de-una-sociedad-criminal/

Ruiz Morales, M. M. (2002). Cultura y territorio en el barrio "la libertad". Centro de Estudios Avanzados en Niñez y Juventud alianza de la Universidad de Manizales y el CINDE (en línea). http://bibliotecavirtual.clacso.org.ar/Colombia/alianza-cinde-umz/2013040 5052115/TM aicolMauricioRuiz.pdf

Salgado, F. (2009). Representaciones sociales acerca de la violencia escolar. REICE, 7(3), 138-152.

Simkin, H., Becerra, G. (2013). El proceso de socialización. Apuntes para su exploración en el campo psicosocial. Ciencia, docencia y tecnología, 24(47), 119-142.

Sirota, R. (1998). L'émergence d'une sociologie de l'enfance: évolution de l'objet, évolution du regard. Éducation et Sociétés (2), 9-33.

UNICEF (2018). Los derechos de la infancia y la adolescencia en México. México: Autor. 
Wright, S. (2007). Young children's meaning-making through drawing and 'telling': Analogies to filmic textual features. Australasian Journal of Early Childhood, 32(4), 37-48.

Zuluaga Gómez, A. (2018). Representaciones sociales construidas sobre el cuerpo femenino por mujeres adolescentes, víctimas de explotación sexual. Revista Facultad Nacional de Salud Pública, 36(1), 75-82. 10.17533/udea.rfnsp.v36n1a09 